\title{
Design and implementation of automated management system based on environmental information of ocean fisheries
}

\author{
Cao DanYang ${ }^{1, a}$, Liu Jie ${ }^{1, b}$, Song XiZhong ${ }^{2}$ and Yang QiuQi ${ }^{1, c}$ \\ ${ }^{1}$ College of Computer Science, North China University of Technology. Beijing, 100043. \\ ${ }^{2}$ College of Information Engineering, Huang Huai University. Henan, 463000. \\ aufocdy@163.com, b413686520@qq.com, ${ }^{\mathrm{c}} 215436027 @ q q . c o m$
}

Keywords: Ocean fisheries, Satellite Data, Automatic Download, Marine monitoring.

\begin{abstract}
Ocean fisheries environmental information includes sea surface temperature, ocean currents, sea surface height, sea surface wind field and so on. To be more efficient, rapid access to environmental information of ocean fisheries, we designed an automatic management system of data acquisition for its complexity of ocean fisheries environmental information.
\end{abstract}

\section{Introduction}

In the study of ocean fisheries' environmental information, basically, the traditional methods take observation and site investigation. Not only spending a lot of manpower and material resources, having slow effect, high cost and operating at fixed point, but also having some difficult to apply to a wide range of monitoring measurements ${ }^{1-2]}$. Meanwhile, totally artificial ways to manage a large number of ocean fisheries' environmental information are very difficult even for the professionals. With the rapid development of modern information technology, improving the traditional methods of monitoring measurement become particularly urgent. Satellite remote sensing technology come into being because of this technical environment. Satellite remote sensing technology could monitor the changes about marine environment in a real-time, synchronous and efficiently way. It could not only monitor the fisheries' environment, analyze the fishing location, determine the fishing season and develop the overseas fishing grounds and new kinds of fishing, but also could estimate the fishery productivity and monitor the fisheries pollution.

The research and application of satellite remote sensing technology began in the 1970s,our nation also started it during that time. And we had two outcomes: (1) Use of satellite remote sensing information research fisheries marine environment; (2) Analysis the correlation between remote sensing information and marine fisheries. From 1982 years, our nation released some charts about fishery sea conditions based on the meteosat images, combined with the water temperature and other fishing condition's measured data. And the charts were popular. After that, the former Soviet Union, Japan, Britain and France also started some work in this area ${ }^{[3]}$.

To download, store and process the Ocean fisheries' environmental information and data efficient, convenient and automatic is an important component of this Automation ocean monitoring system. When getting fishery environmental information, professionals need to spend a lot of time and energy for daily data download and data management, and usually these are more cyclical work. The more important thing is the data we need to use are becoming more and bigger because of the improvement of this project. Professionals will also face more onerous tasks; it will not only cause a lot of waste of human and material resources, but also a serious impediment to productivity.

This article designed the management system of automated download ocean fisheries' environmental information. This system can be flexibly applied to download the ocean fisheries' environmental information, not only to avoid the cyclical work of professionals, but also the efficient downloading, processing and management for the information.

\section{Composition and design of system}

The automated management systems which based on the Ocean fisheries' environmental 
information automate downloading choose the ocean fisheries' environmental data as the main source of information. This system can automatically download data information, manage data and process data. The main purpose of this system is to provide Man-machine interactive software systems for Professional and technical personnel who study the satellite remote sensing applications in Oceanic. The system has a high degree of automation, information processing powerful features, including four sub-systems: Downloading multi-source data subsystem; processing multi-source data subsystem; the configuration and monitoring subsystem; the task scheduling subsystem. As shown in Fig.1:

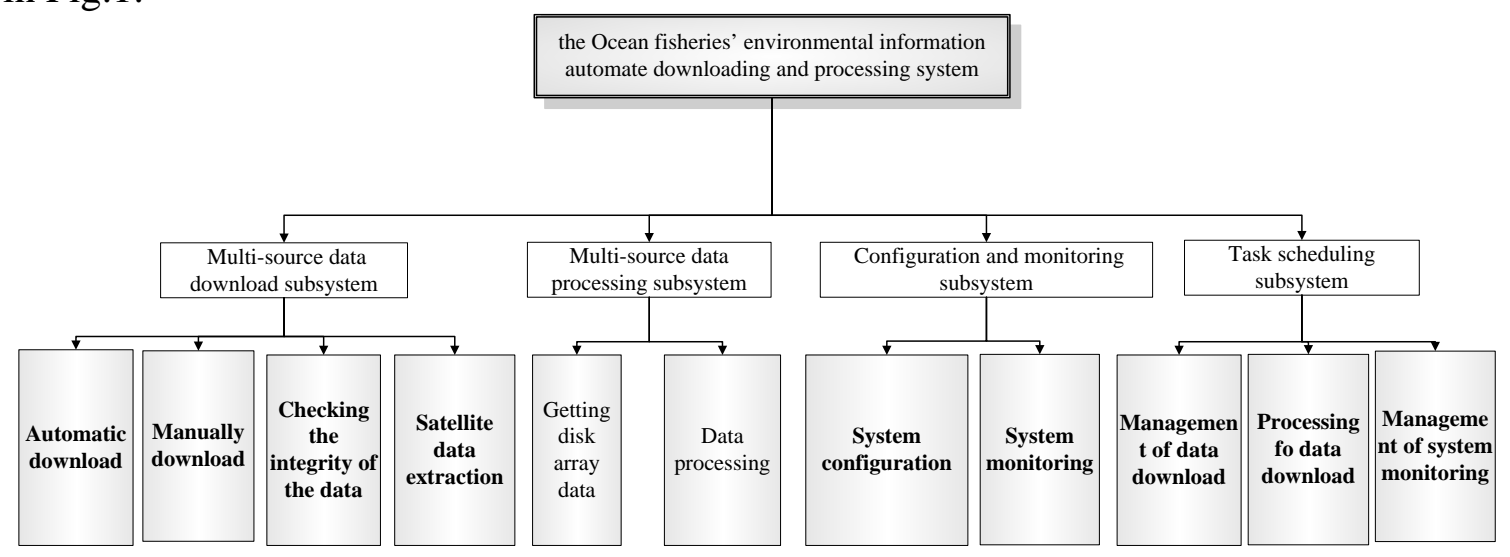

Fig.1.System components

\section{The design of subsystem}

This system uses the idea of modular transverse design. Each module can separate their respective functions, and the corresponding independent external interface, just with the input of a few parameters; each module could communicate with each other. And because of this, it could reduces the coupling between modules in large part and improve the reliability of system.

(1).The design of downloading multi-source data subsystem

Multi-source data processing subsystem is the core module of the entire application system. It is primarily responsible for automatically download tasks of satellite data from different data sources such as FTP, HTTP, EMAIL, customized web pages. It also includes the preparation of relatively independent modules for different data sources as well as fault-tolerant of data processing and completeness checks which provide reliable data when the next data processing. Multi-source data download phase focuses on the data interactive between the automated processing system and data source systems (including FTP, HTTP and customized web pages). This modules need to deal with this issue is how to integrate data from different information sources into one application system.

(2).The design of processing multi-source data subsystem

Multi-source data processing subsystem is external output interface of the entire system. After the automatic download, the processed ocean environment data are distributed to different sites, providing professional and technical personnel for analysis and processing. And its output is the final output of the entire system. The data that wo get from downloading multi-source data subsystem have different format because of the diversity of ocean fisheries and the complexity of data sources of environmental information. This would require a special data format conversion and statistical classification about the converted data.

During the download process from multi-source data, there may be two abnormal data. One abnormal data is caused by the automatically downloaded system's self- restrictions. Such abnormal data are often due to software applications have a variety of unforeseen anomalies, such as network bandwidth limitations, the system of human configuration errors and so the server's exceptions, all of these are uncontrollable situation by people, and these situation could make the download task interrupted in the middle and show some inconsistent condition compare with the original data. As the second abnormal data, they are also uncontrollable situation by people. Because satellite data collection itself is an optical sensor, data acquisition is affected by ocean light conditions, so during 
the data collection process, the day data should be automatically filtered out.

(3).The configuration and monitoring subsystem

The configuration and monitoring subsystem is the foundation that the whole system of foreign expansion. It is mainly responsible for the data node download, the data processing program and the configuration of target file path. Mainly for the increase, delete, modify, query and other operation to the configuration, match different ways to download. So that business processes can be unified configuration management and each business unit can work together.

The monitoring subsystem could be real-time monitoring the case of downloading various types of data, including operating status of automatically downloaded, manually download, data processing and some other programs. When there are some abnormal situations, it could flexible feedback the information to the task scheduling subsystem and staffs.

(4).The task scheduling subsystem

The task scheduling subsystem is the hub of the whole system. These subsystems make multi-source data download and multivariate data processing subsystem divided into different sub-modules, unified management of these sub-modules; assume the coordination and scheduling of work between these sub-modules, full consideration Fault tolerance and completeness between these sub-modules.

The task scheduling system has two ways: automatic scheduling and manually scheduling. According to the date time, latitude and other parameters, automatic scheduling could be timing generating new tasks everyday without people involved and call automatically download and processing sub-module, then, send the processed data to the specified user. At the same time, saving the auto task downloaded log so that people can review it. As for the manually scheduling, we should mainly consider some abnormal data such as lack and leakage. In that case, we need add these data by staffs, then, the whole progress would be same with the automatic scheduling.

\section{Analysis and description of Systems Business Process}

System uses a hierarchical design of the longitudinal to avoid each logical thinking tightly intertwined, so that we can achieve the purpose of separation of concerns, loose coupling, multiplexing logic and the standard definition ${ }^{[3-5]}$.

The system of downloading and processing information and data of Ocean fisheries' environmental have a clear processing, see in Fig.2.

As we can see in the above figure, the overall flow chart systems have the main business processes and the business process support processes. The overall system consists of four sub-processes and two types of flow process. Four sub-processes are downloading multi-source data subsystem, processing multi-source data subsystem, the configuration and monitoring subsystem and the task scheduling subsystem. Two types of flow process are automatic processing and HCI processing.

Fig.2 shows that automatic processing has four phases: System Configuration, Data Download, Data processing, Data Extraction. Next, according to the type of processing, we would introduce four treatments types of business processes.

1) Base on the automatic processing

(1) Configuring the data sources and the file path

(2) Task scheduling subsystem generate download task automatically

(3) Detect the integrity of data

(4) Multi-source data processing subsystem process the downloaded data

(5) Generate the target data

(6) Save the target data into the disk array

2) Base on the HCI processing

(1) According to the actual needs, user selects the data source which needs to be downloaded

(2) Detect the integrity of data

(3) Multi-source data processing subsystem process the downloaded data

(4) Generate the target data 
(5) Save the target data into the disk array

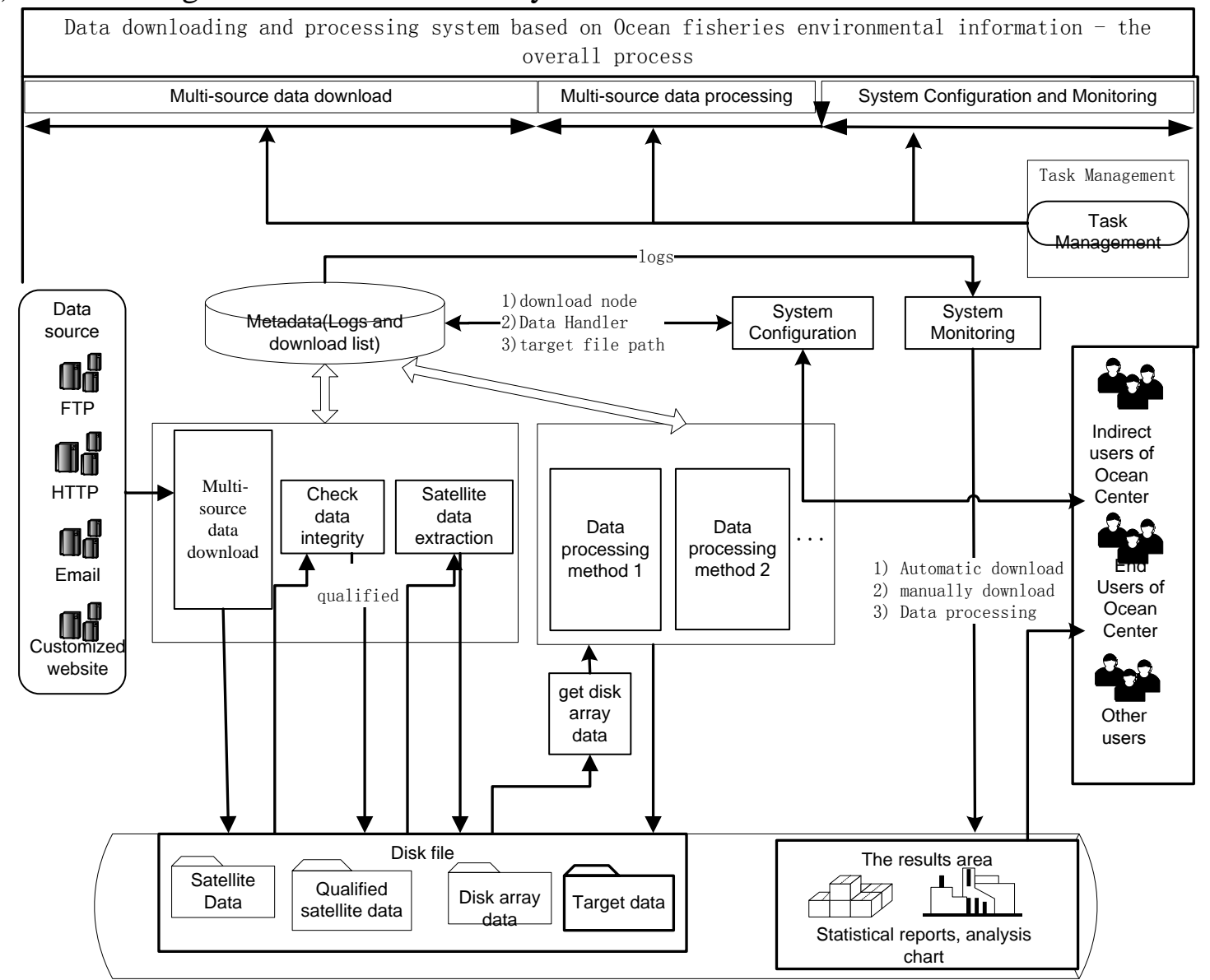

Fig.2. System process flow chart
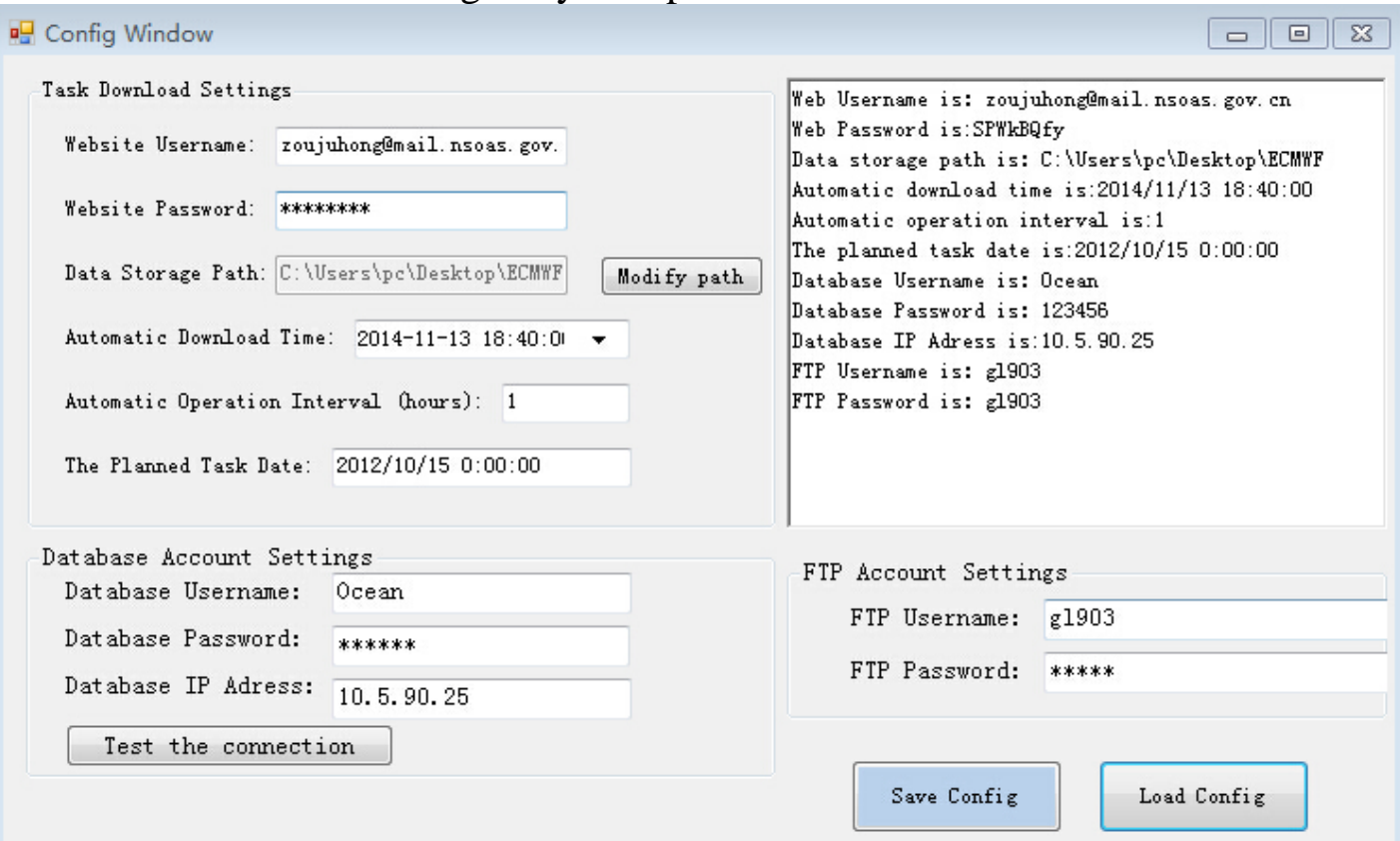

(After modified configuration item, please click on the Save button!)

Figure 3.Configuration diagram

\section{Implementation of Ocean Fisheries Environment Information System}

Due to the ocean environmental information data come from different data sources such as FTP, HTTP and Customized website. So according to the characteristics of the data source we have 
different downloader, and every downloader could independent run themselves.

The data that download by this downloader need to be checking their size. Finally, save the right data into the disk array. Besides that, we also provide manually download way so that this system can be Flexible and robust. Here is part of the achievement system.

As shown in figure 3, we use class FormConfig to finish the function that configuring the system.

As shown in Figure 4, we use class FormAuto and class FormTask in window Task Management to achieve real-time data monitoring and downloading capabilities.

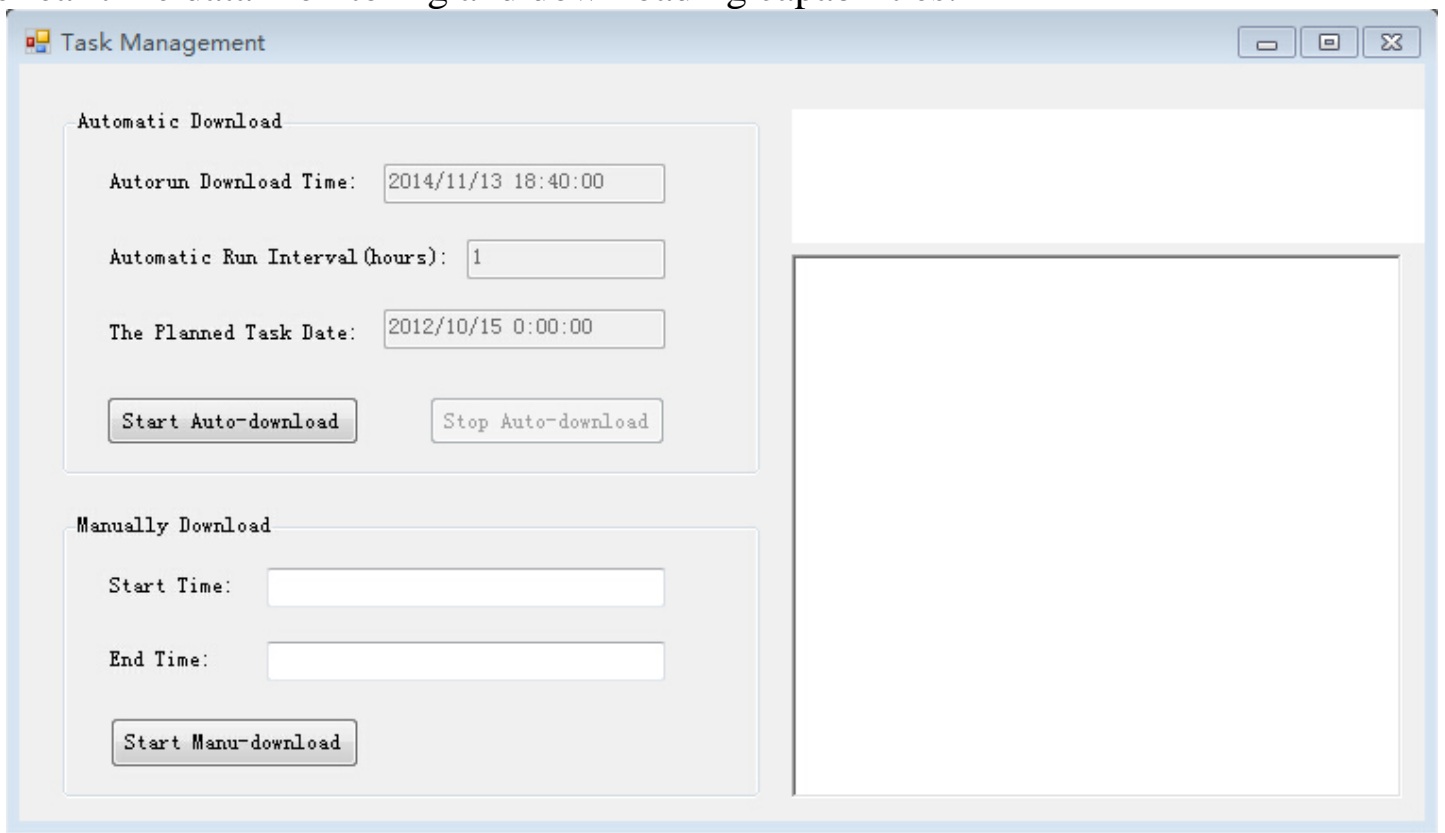

Figure 4. Task planning and monitoring diagram

There is an example that downloads data which come from 2012/10/24 to 2012/10/25, at the first time; we should configure some information such as the download path, user name and password. Then according the time, system will find if there have some data which need to be downloaded. If there have, the system will generate the new task and use some downloader such as ftp, http and email to automated download data. Finally, system sends the processed data to the specific user.

\section{Problems}

These system have many different data source, because of that, there might have some unforeseen situation. For instance, customized website, due to minor changes of web pages might make the downloader do not work. At the same time, different data resource have different data, and how to efficient statistical these data are still a problem we need to fix.

\section{Conclusions}

The system of automatic download environment information of ocean fishing grounds could automatic download these data. In the absence of intervention, automatically completes the download of data and processing, and achieve a unified management of data. Because of that, we could improve the speed of getting the information of fishing ground and improve the user experience.

\section{Acknowledgements}

This paper is supported by Beijing Natural Science Foundation (NO.4144073), Supported by Research Fund of North China University of Technology, Supported by North China University of Technology Science Foundation, and the Central Support the Local Special (NO.PXM2014_014212_000097). 


\section{References}

[1] Xingbao Li, Fang Hou. Automatic C/S-Based Meteorological Data Downloading System. Meteorological science and technology, 2010, 38(5):609-612.

[2] Cuilin Kuang, Rong'an Xie, Yantao Wang, Wujiao Dai. Software design and implementation for IGS data antomatic batch download. Geotechnical Investigation \& Surveying, 2013(7):72-75.

[3] Xinjiang Zheng, Tianxi Fan. Meteorological satellite remote sensing applications in marine fisheries. China Aerospace, 1997(3):3-4

[4] Feng Li, Fanlin Yang, Yunwei Li, Huijuan Zhang. Automatic GPS Data Downloading System based on Bemese5.0.Mine Survering, 2010(6):27-30.

[5] Jesse Liberty, Brian Macdonald. C\# 3. 0 Study Guide. Beijing, Post \& Telecom Press, 2010. 